\title{
Role of Affective-Emotional Temperament in Anticipation of Post-Traumatic Stress Disorder Symptoms in Maimed Individuals Caused by Mine Explosion
}

\section{ART ICLE INF O}

\section{Article Type}

Descriptive Study

\section{Authors}

Shafiei M. ${ }^{1} P h D$,

Basharpoor S. ${ }^{2} P h D$,

Heidarirad H.* MSC

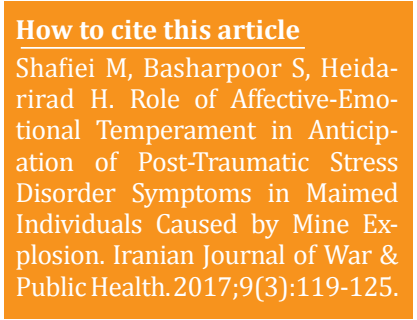

*Psychology Department, Educational Sciences \& Psychology Faculty, University of Mohaghegh Ardabili, Ardabil, Iran

${ }^{1}$ Psychology Department, Literature \& Humanities Faculty, Lorestan University, Khoramabad, Iran ${ }^{2}$ Psychology Department, Educational Sciences \& Psychology Faculty, University of Mohaghegh Ardabili, Ardabil, Iran

\section{Correspondence}

Address: Educational Sciences \& Psychology Faculty, University of Mohaghegh Ardabili, Daneshgah Street, Ardabil, Iran

Phone: +98 (83) 43226702

Fax: +98 (83) 43226702

hadeesheidarirad@gmail.com

\section{Article History}

Received: January 31, 2017

Accepted: March 12, 2017

ePublished: July 27, 2017

\section{A B S T R A C T}

Aims Post-Traumatic Stress Disorder (PTSD), is one of the most common, chronic and debilitating psychiatric disorders that occurs after encountering a traumatic event. Studies show that psychological responses from exposure to harmful events are influenced by the affective-emotional temperament of individuals. Therefore, the purpose of this study was to determine the role of affective-emotional temperament in predicting the symptoms of posttraumatic stress disorder in maiamed individuals caused by mine explosion.

Instrument \& Methods This descriptive correlation study was performed on 100 people among all the people who had suffered a defect in the mine during the second half of 2016 under the protection of the martyr foundation of Gilan-e-Qarb City, Iran. The research instrument was a structured clinical interview, the Mississippi post-traumatic stress disorder scale, and the Affective and Emotional Composite Temperament (AFECT). The collected data were analyzed using Pearson correlation coefficient and multiple linear regression analysis using step by step method in SPSS 20 software.

Findings In sum, affective temperament components explained 51\% and emotional temperament components explained $32 \%$ of variance of symptoms of post-traumatic stress disorder $(\mathrm{p}<0.05)$.

Conclusion Affective and emotional nature can contribute to defect in how people respond to traumatic events and symptoms of post-traumatic stress disorder in maimed individuals caused by mine blast.

\section{Keywords Temperament; Stress Disorder, Post-Traumatic; Amputation; Explosion}

\section{I T A T I O N L INKS}

[1] The prevalence of posttraumatic stress disorder among American Indian veterans ... [2] Predictors of posttraumatic stress in ... [3] A randomized controlled trial of the effectiveness of writing as intervention for traumatic injury patients at risk of developing ... [4] Diagnostic and statistical manual of ... [5] Predictive factors of chronic post-traumatic stress disorder ... [6] Prevalence of post traumatic stress disorder and ... [7] Study of health related quality of life in posttraumatic stress ... [8] Temperament and its role in developmental ... [9] The comparison of temperament, character, and ... [10] Evaluation of personality dimensions using the ... [11] The affective and emotional composite temperament (AFECT) model and scale ... [12] Suggestive linkage of a chromosomal locus on 18p11 to ... [13] The influence of affective temperaments and psychopathological traits on the definition of bipolar disorder subtypes ... [14] Affective Dispositions and PTSD Symptom Clusters in ... [15] A pro-posed model of Alzheimer's dementia and PTSD ... [16] MAssessing a five factor model of PTSD: Is dysphoric arousal a unique PTSD construct showing differential relationships ... [17] Personality heterogeneity in PTSD: Distinct temperament and interpersonal ... [18] New DSM-5 maladaptive symptoms in PTSD: Gender differences and correlations with mood spectrum symptoms in a sample of high school students ... [19] Differential effects of anxiety sensitivity components in the relation between emotional ... [20] Comorbidity of PTSD in anxiety and depressive disorders .. [21] A psycho physiological investigation of emotion ... [22] Impaired right inferior frontal gyrus response to contextual cues in ... [23] Risk and resiliency in posttraumatic stress disorder ... [24] Work volition among U.S. veterans ... [25] The theorical and practical fundamental of research in social and ... [26] Mississippi scalefor combay ... [27] Evaluating reliability and validity of the Mississippi scale for posttraumatic ... [28] Hilgard's introduction ... [29] The Effects of attention training on health [30] Children's and adolescents' aggressive behavior in context ... 
جمله ملاكهاى تشخيصى اصلى براى PTSD بوده و خطر ابتلا به

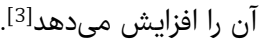

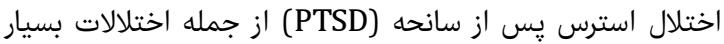

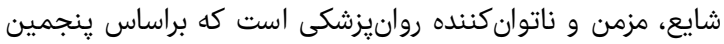

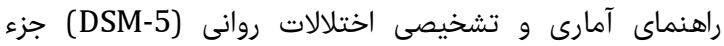

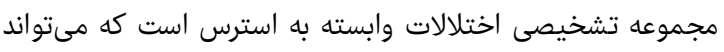

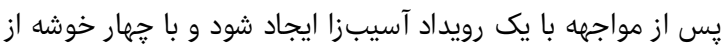

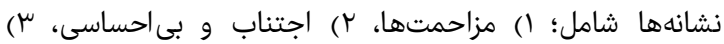

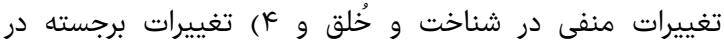

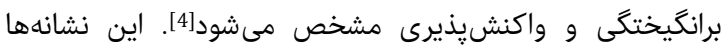

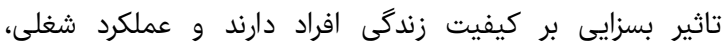

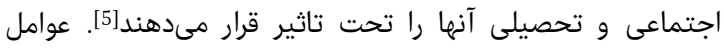

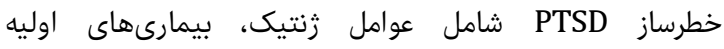

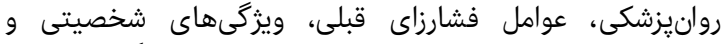

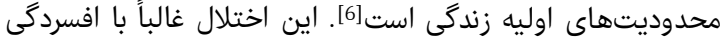

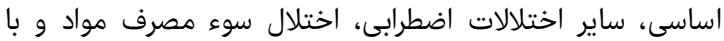

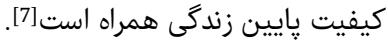

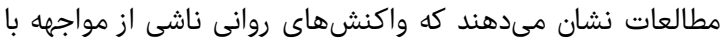

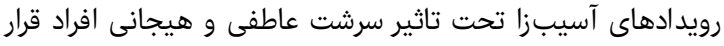

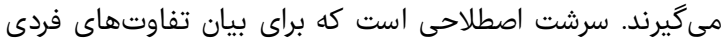

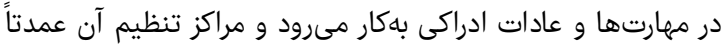

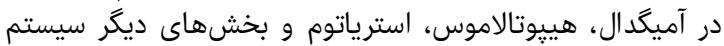

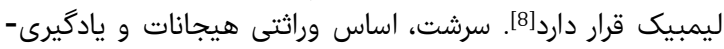

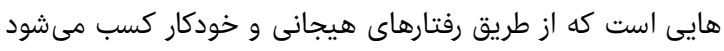

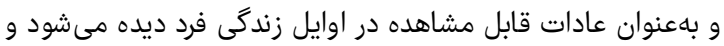

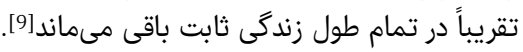

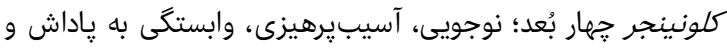

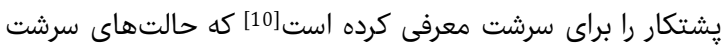

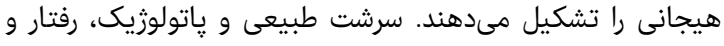

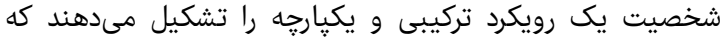

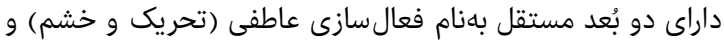

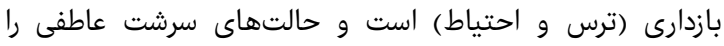

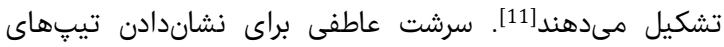

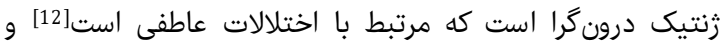

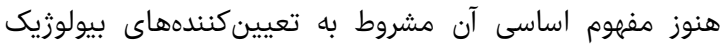

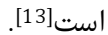
با ادغام مفاهيم سرشت هيجانى و سرشت عاطفى مدل مركب

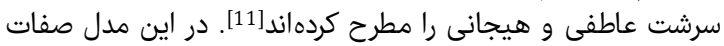

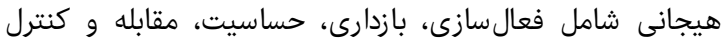

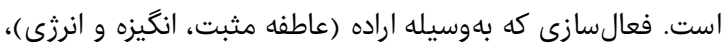

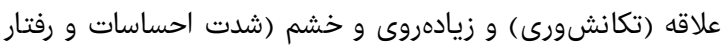

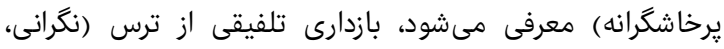

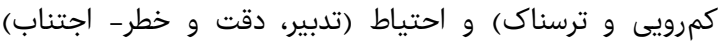

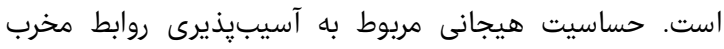

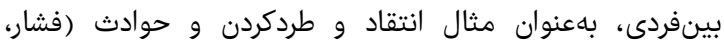

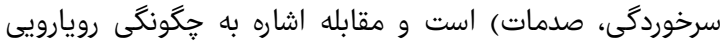

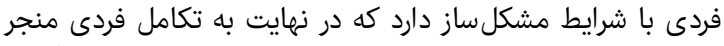

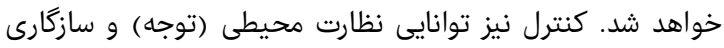

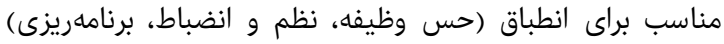

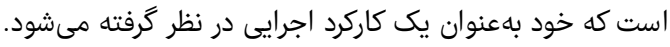

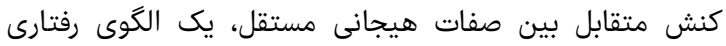

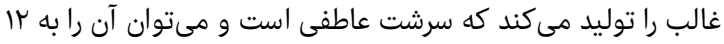

دوره 9، شماره ץ، تابستان عوسا
نقش سرشت عاطفى -هيجانى در ييشبينى علايم

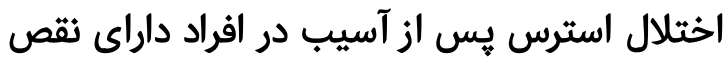
عضو در اثر انفجار مين

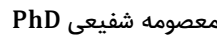
كروه روانشناسى، دانشكده ادبيات و علوم انسانى، دانشگاه لرستان، خرمآباد، ايران

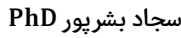
كروه روانشناسى، دانشكده علوم تربيتى و روان شناسى، دانشكاه محقق اردبيلى،

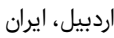

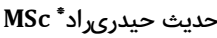

كرّه روان شيناسى، دانشكده علوم تربيتى و روان شناسى، دانشكاه محقق اردبيلى، اردبيل، ايران

جكيده

اهداف: اختلال استرس يس از سانحه (PTSD) از جمله اختلالات بسيار

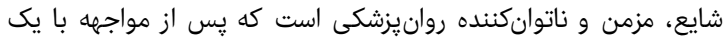

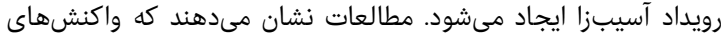

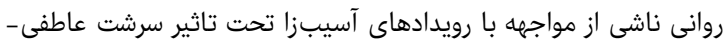

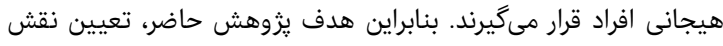

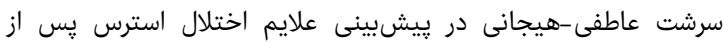

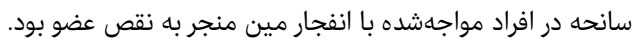

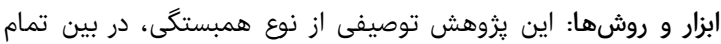

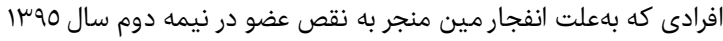

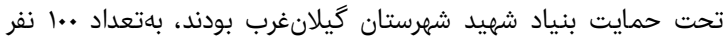

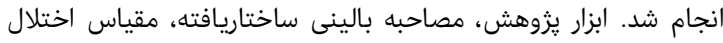

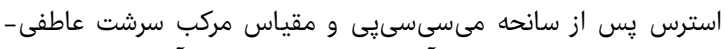

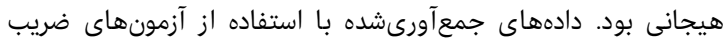

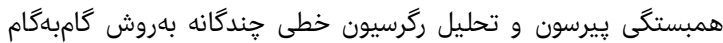

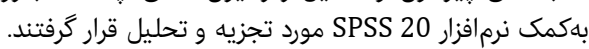

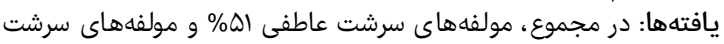

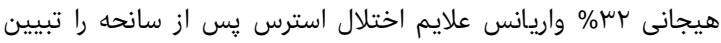

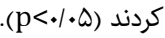

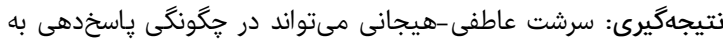

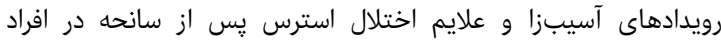

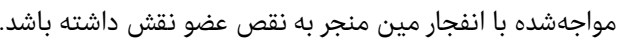

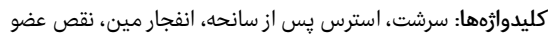

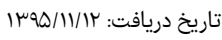

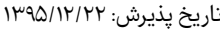

"نويسنده مسئول: hadeesheidarirad@gmail.com

مقدمه مقان

ساليان متمادى است كه متخصصان بهداشت روانى تلاش مى إكنند

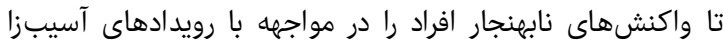

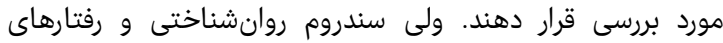

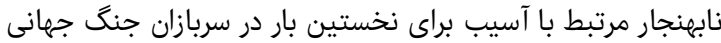

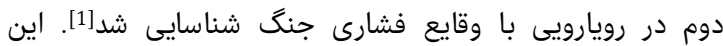

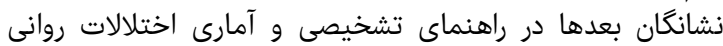

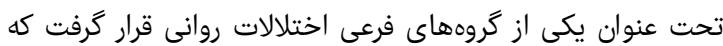

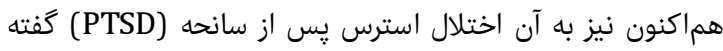

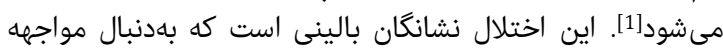

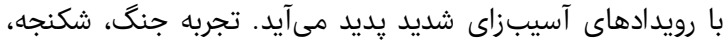

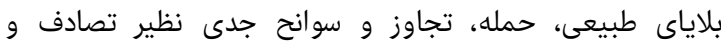

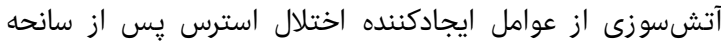

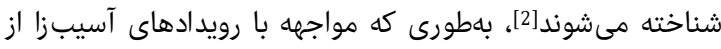

فصل نامه علمى - يزووهشى طب جانباز 


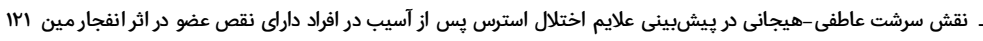

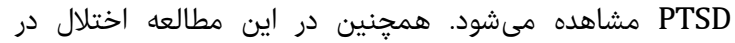

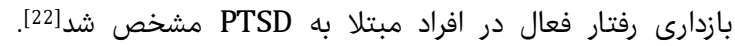

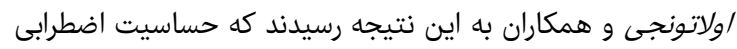

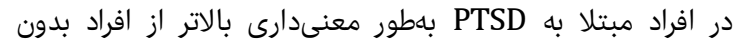
PTSD

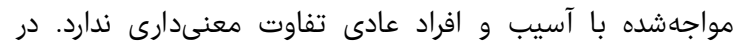

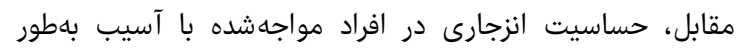

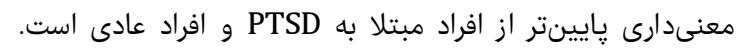

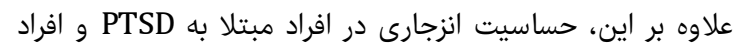

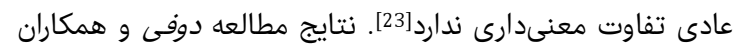

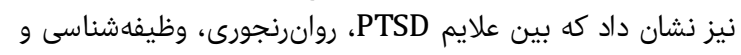
اراده ارتباط وجود دارد[24].

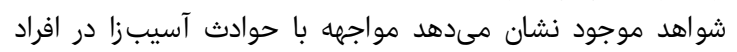

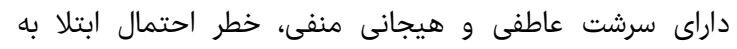

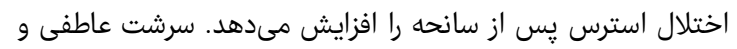

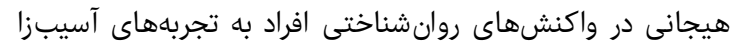

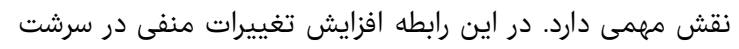

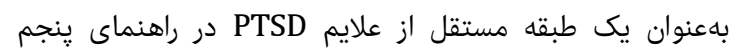

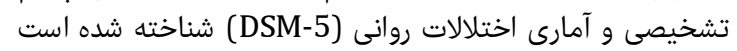

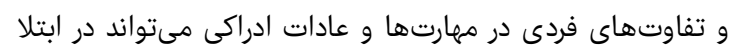

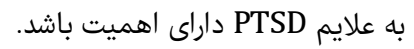

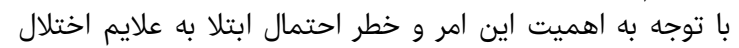

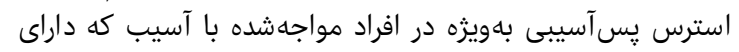

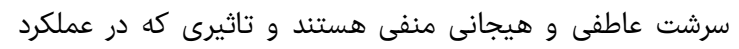

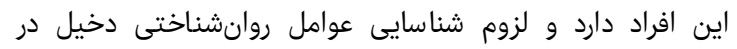

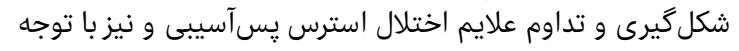

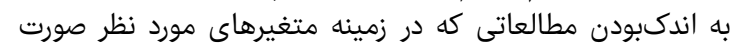

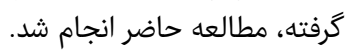

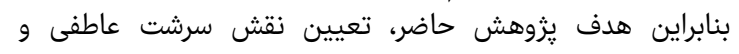

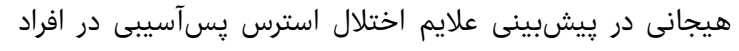

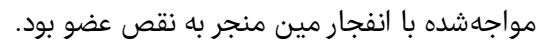

\section{ابزار و روشها}

اين يزوهش توصيفى از نوع همبستگى در بين تمام افرادى كه

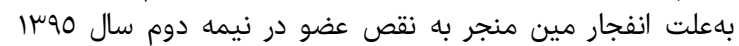

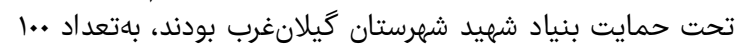

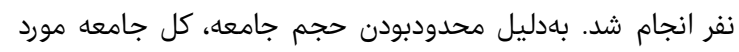

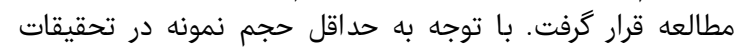

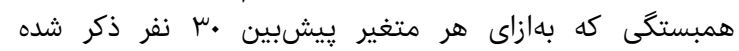

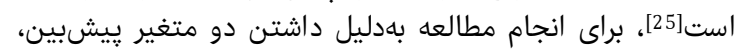

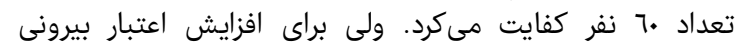

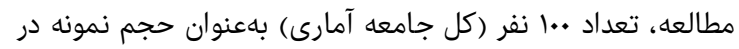

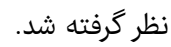

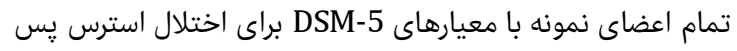

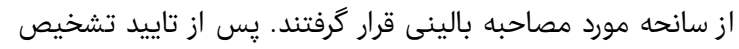

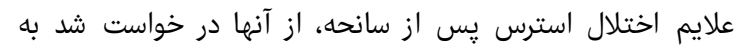

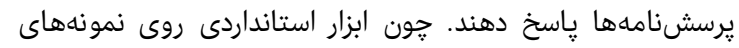

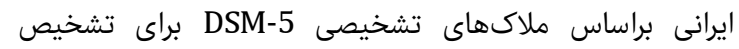

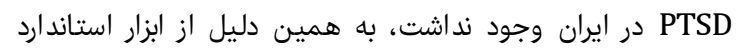

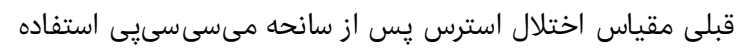

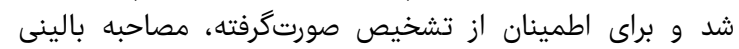

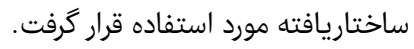

Iranian Journal of War and Public Health
نوع؛ افسرده، مضطرب، بىتفاوت (تيڤهاى درونى)، ادوارىخوى،

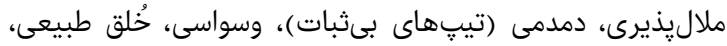

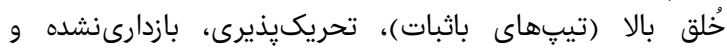

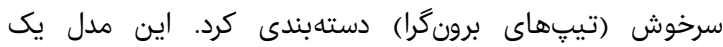

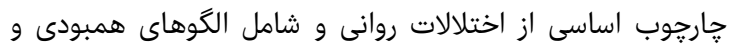

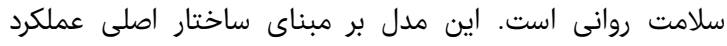

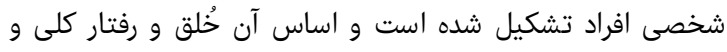

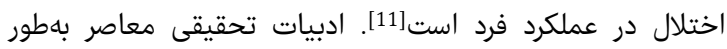

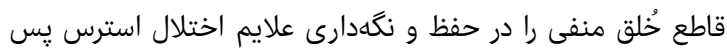

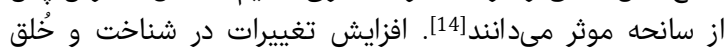

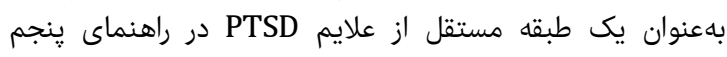
تشخيصى و آمارى اختلالات روانى (DSM-5) شناخته شده است است.

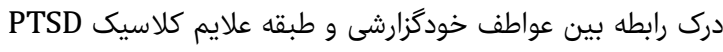

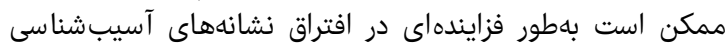

روانى در افراد مواجه شده با آسيب موثر باشد الشئ.

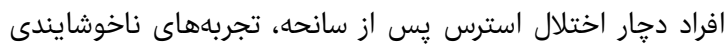

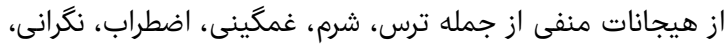

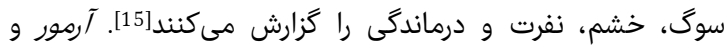

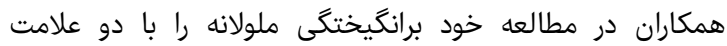

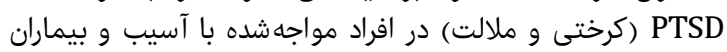

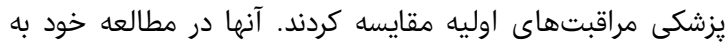

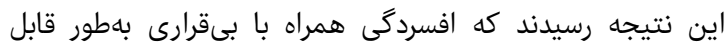

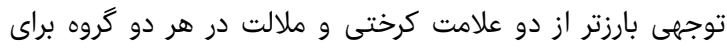

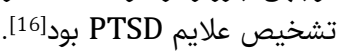

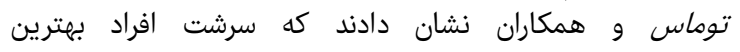

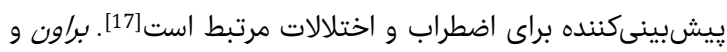

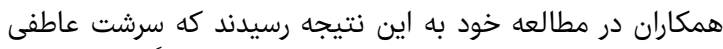

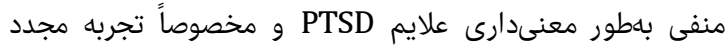

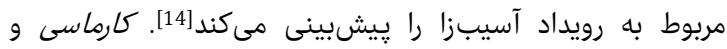

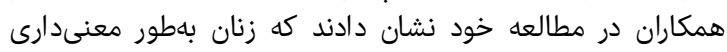

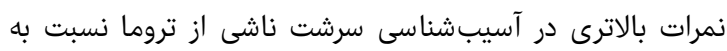

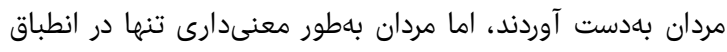

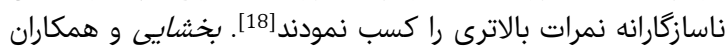

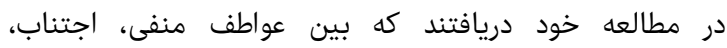

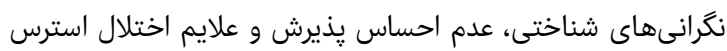

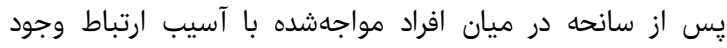

دارد [19].

در يزوهش اسبينهون و همكاران اين نتايج بهدست آمد كه با

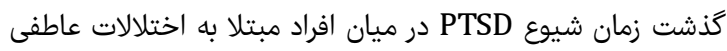

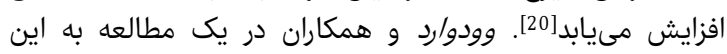
نتيجه رسيدند كه افراد مبتلا به وادواله

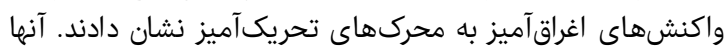

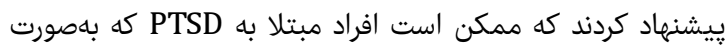

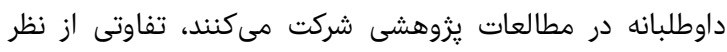

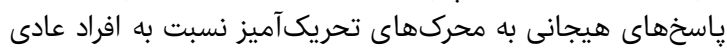

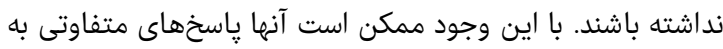

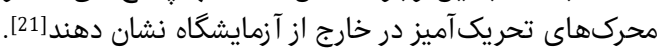

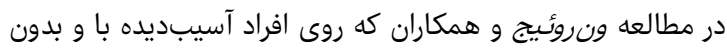

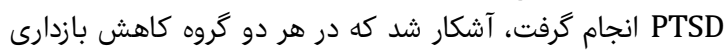

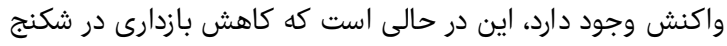

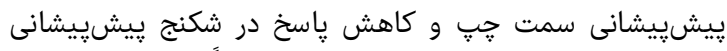

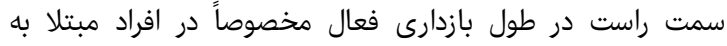

Volume 9, Issue 3, Summer 2017 


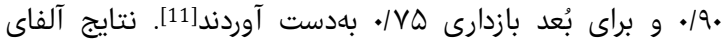

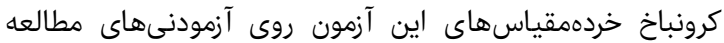

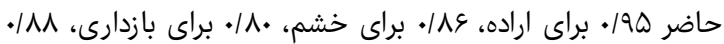

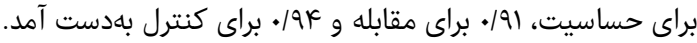

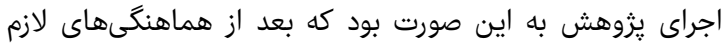

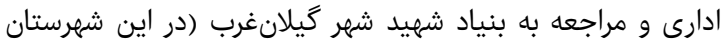

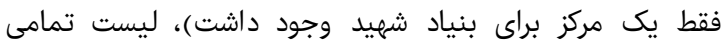

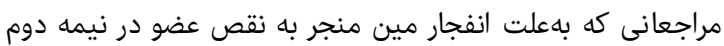

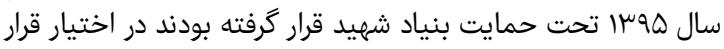

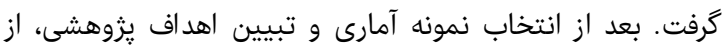

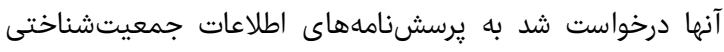

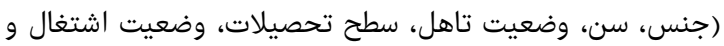

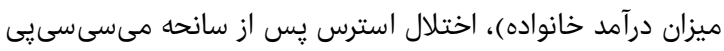

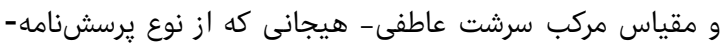

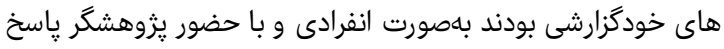
دهند.

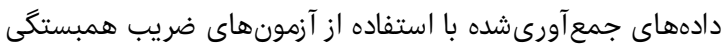

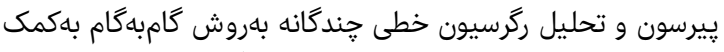
نرم افزار SPSS 20 مورد تجزيه و تحليل قرنيل ترار كرفتند.

\section{يافتهها}

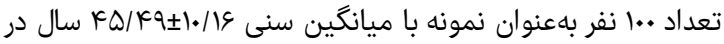

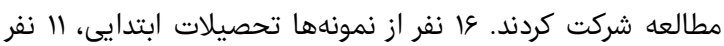

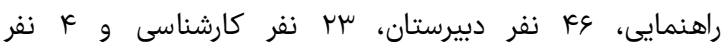

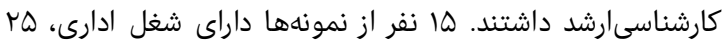
نفر شغل آزاد و •9 نفر بيكار بودند.

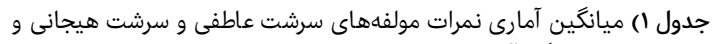

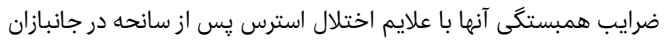

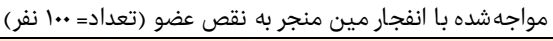

\begin{tabular}{|c|c|c|}
\hline ضريب همبستگى & ميانگين & متغيرها \\
\hline & & سرشت عاطفى \\
\hline . $/ \Delta r^{* * *}$ & $r / F \Delta \pm I / F V$ & افسرده \\
\hline.$/ k K^{\circ}$ & $r / V \cdot \pm 1 / \mu \wedge$ & مضطرب \\
\hline.$/ \mu k^{* *}$ & $r / \mu F \pm 1 / \mu F$ & بىتفاوت \\
\hline.$/ k \mu a$ & $r / V \Delta \pm I / K$. & ادوارىخوى \\
\hline$\cdot \mid \Delta K^{\circ \circ}$ & $r / F \mu_{ \pm} I / T K$ & ملال يذيرى \\
\hline.$/ \& q^{* *}$ & $r / \Delta s \pm 1 / \mu$. & دمدمى \\
\hline . & $\mu / \mid \Lambda \pm 1 / \mu \Lambda$ & وسواسى \\
\hline.$/ 1$ & $\mu / r \Delta \pm 1 / \mu \mu$ & خلق طبيعى \\
\hline.$/ 1$ & $\mu / r \Delta \pm 1 / F F$ & خلق بالا \\
\hline.$/ r V^{*}$ & $\mu / . . \pm 1 / \mu \Delta$ & تحريكيذير \\
\hline.$/ K Q^{\circ \circ}$ & $r / \Delta V \pm I / \mu$. & بازدارىنشده \\
\hline \multirow[t]{2}{*}{.$/ 4 V^{s *}$} & $r / \Lambda \Lambda \pm I / r V$ & سرخوش \\
\hline & & سرشت هيجانى \\
\hline.$- / \mu \omega^{* *}$ & $\mu r / \cdot V \pm \| l / \kappa q$ & 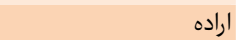 \\
\hline$\cdot|r|^{\circ}$ & $\mu \mid / / \kappa_{ \pm} \wedge / \wedge \varepsilon$ & خشم \\
\hline $.1 \cdot 0$ & $\mu_{1} / V k \pm N / \cdot q$ & بازدارى \\
\hline$. / \mu k=$ & $\mu I / F V \pm q / V r$ & حساسيت \\
\hline.$- \% v$ & $\mu \mu / . . \pm \| l / r$. & مقابله \\
\hline.$- / 1 \mu$ & $\mu r / K \cdot \pm \mid r / 1$. & كنترل \\
\hline
\end{tabular}

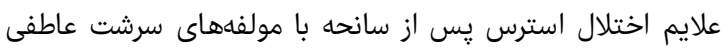

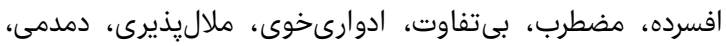

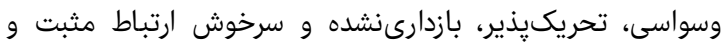

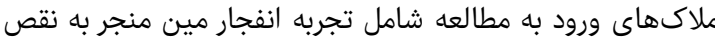

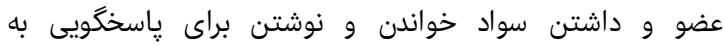

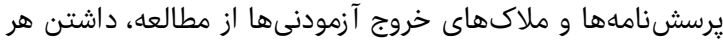

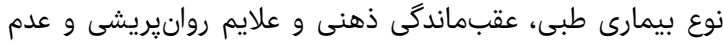

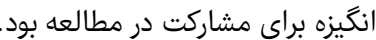

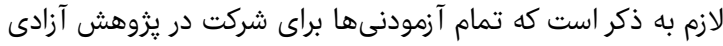

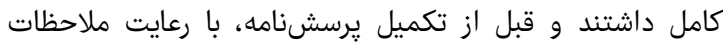

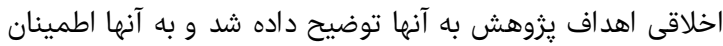

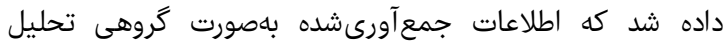

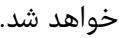
ابزار يزوهش، مصاحبه بالينى ساختاريافته، مقياس اختلال استرس

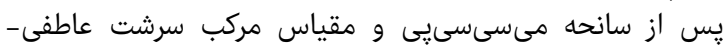

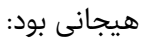
ا- مصاحبه بالينى ساختاريافته: بهمنظور بررسى اعتبار و تاييد

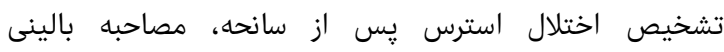

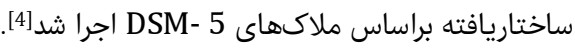

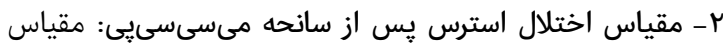

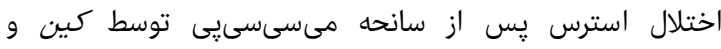

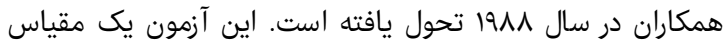

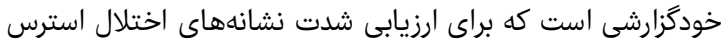

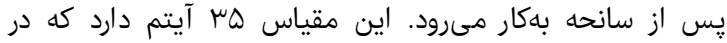

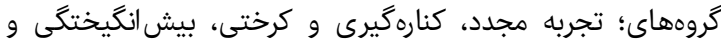

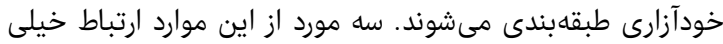

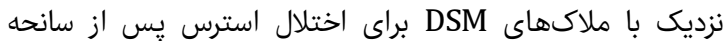

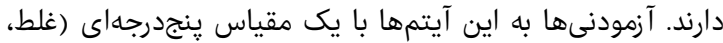

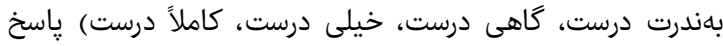

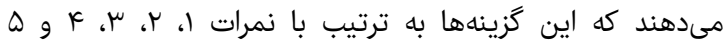

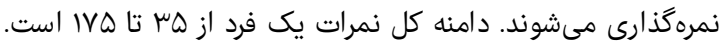

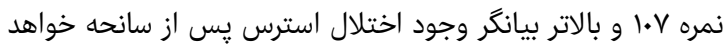

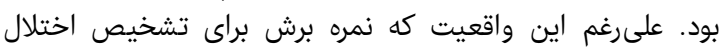

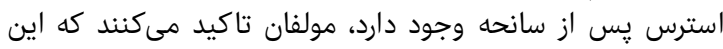

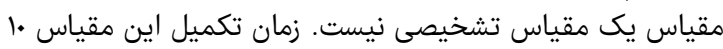

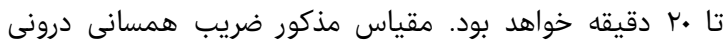

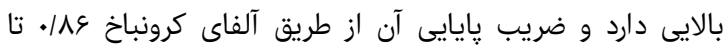

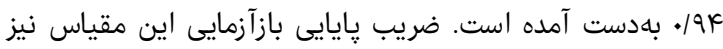

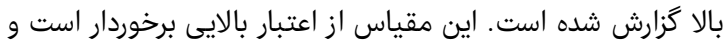

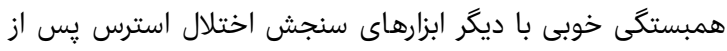

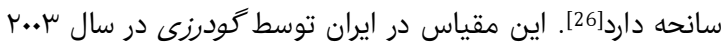

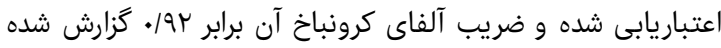

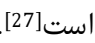

ץ- مقياس مركب سرشت عاطفى- هيجانى (AFECT): مقياس

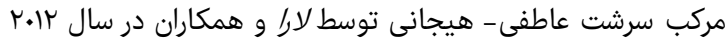

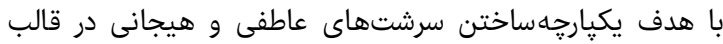

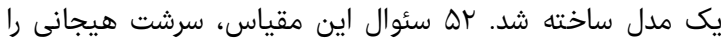

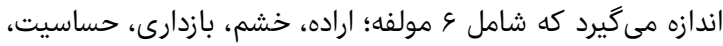

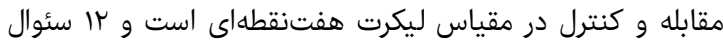

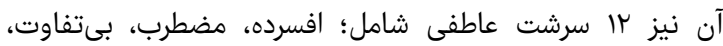

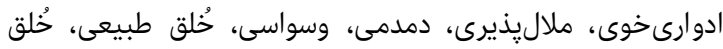

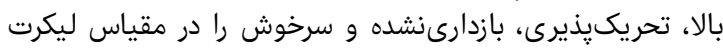

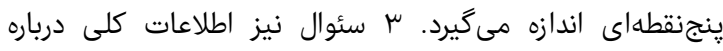

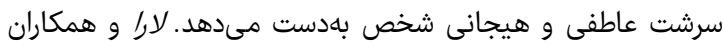

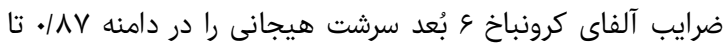




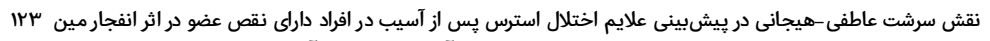

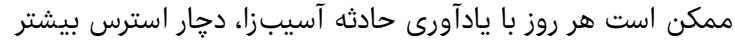

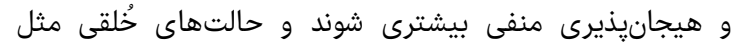

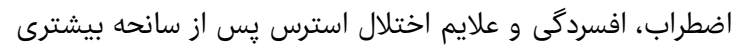

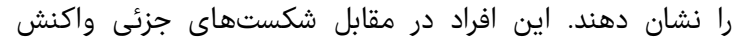

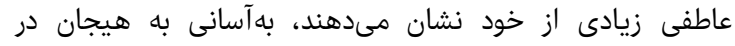

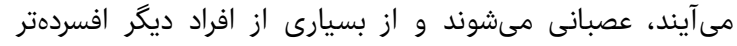

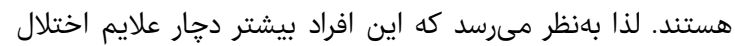
استرس يس از سانحه شوند.

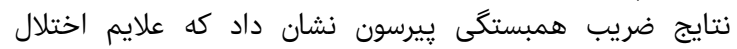

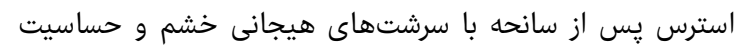

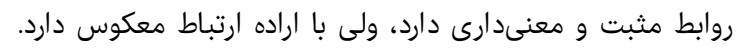

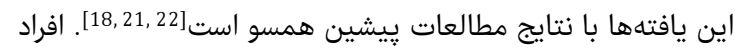

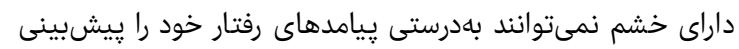

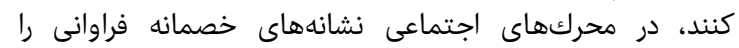

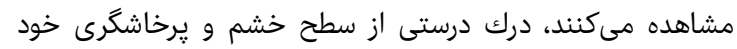

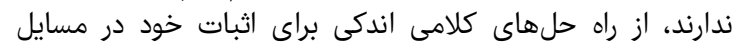

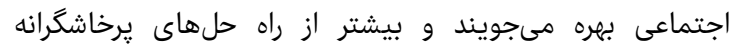

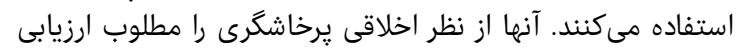

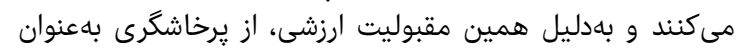

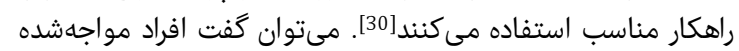

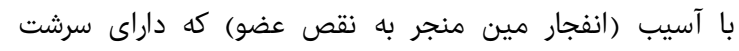

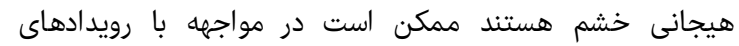

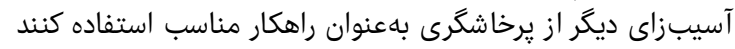

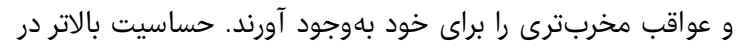

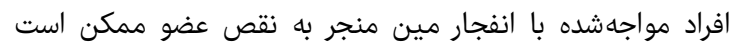

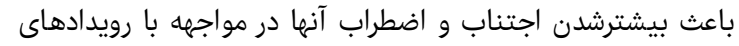

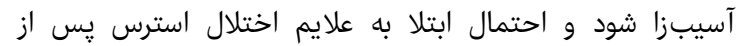

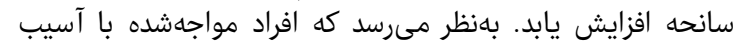

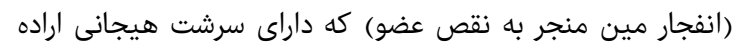

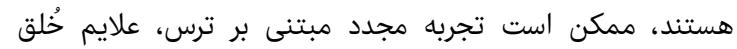

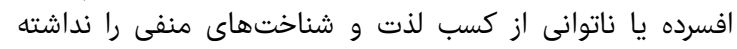

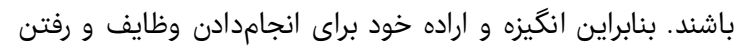

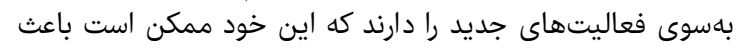

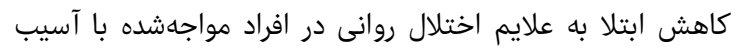

شود.

نتايج اين مطالعه نشان داد كه هر جه مئه ميزان هيجانات منفى در

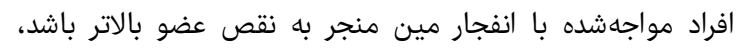

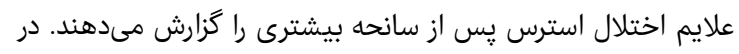

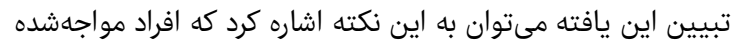

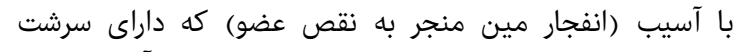

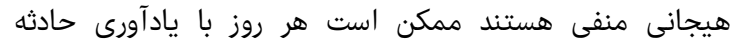

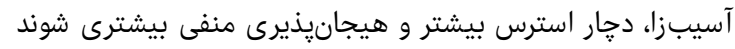

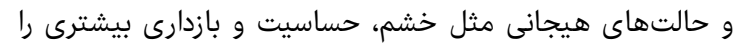

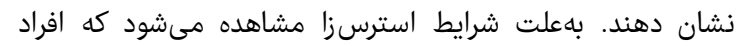

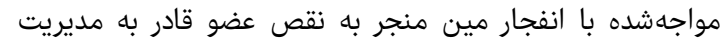

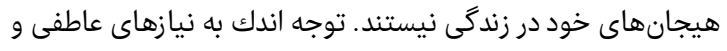

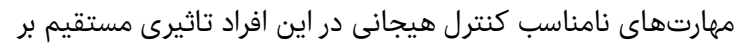

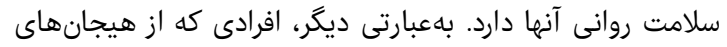

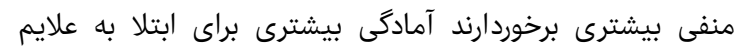

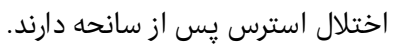

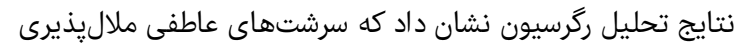

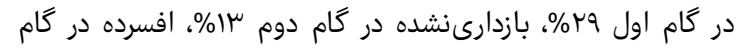

معنىدارى داشت. همجنين علايم اختلال استرس يس از سانحه با

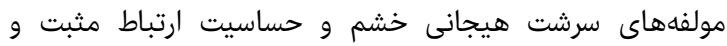

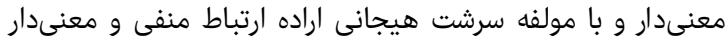

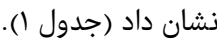

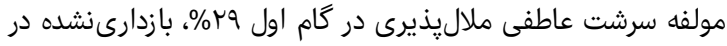

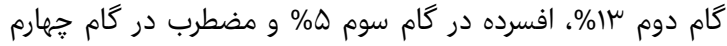

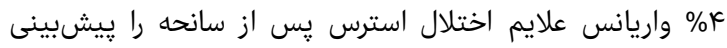

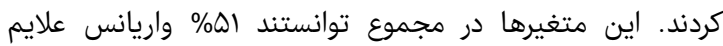

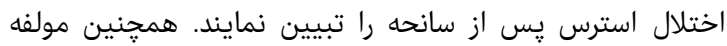

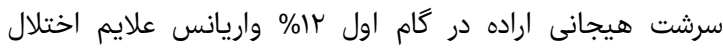

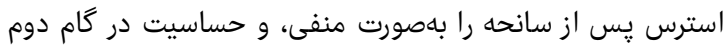

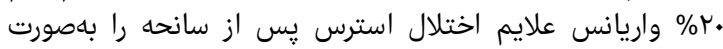

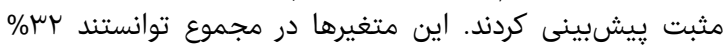

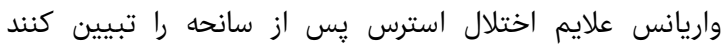

.$(p<\cdot / \bullet \bowtie)$

بحث نتايث ضريب همبستخى بيرسون نشان داد كه علايم اختلال

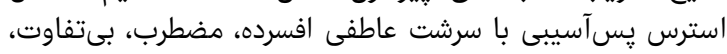

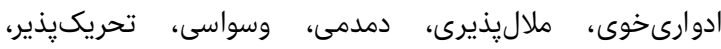

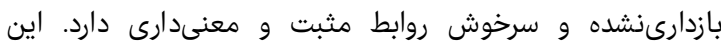
يافتهها با نتايج مطالعات يُشيشين همسو است

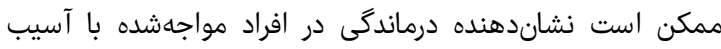

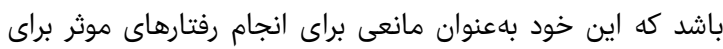

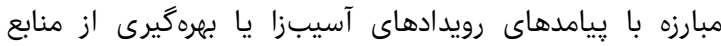

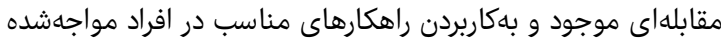

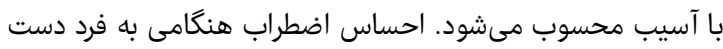

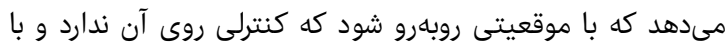

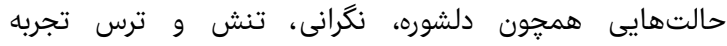

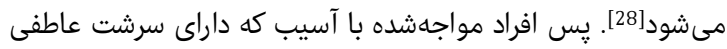

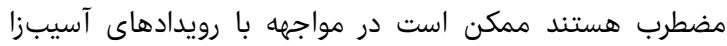

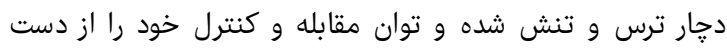

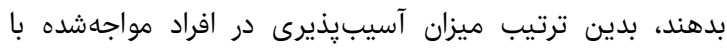

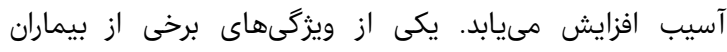

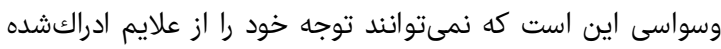

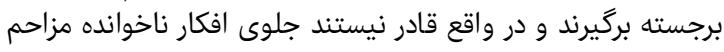

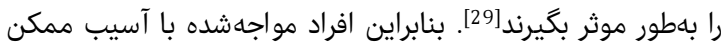

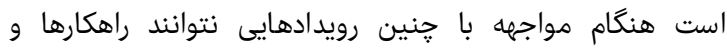

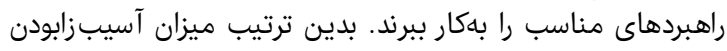
رويداد افزايش مىيابد.

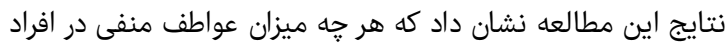

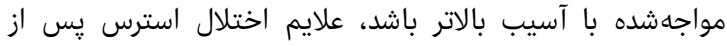

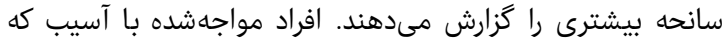

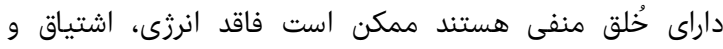

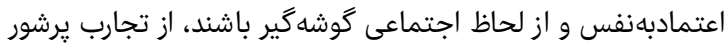

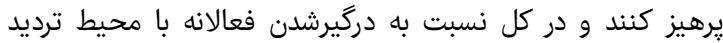

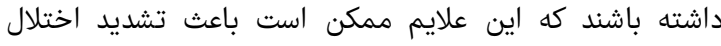

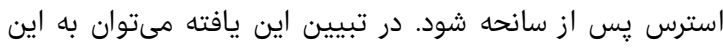

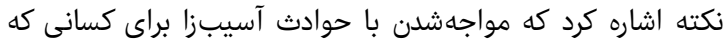

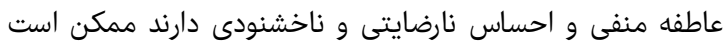

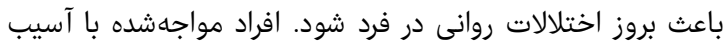

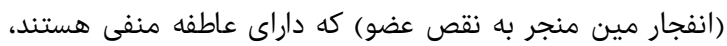


متغيرهاى ييشبين اين مطالعه ييشنهاد مىشود. با توجه به

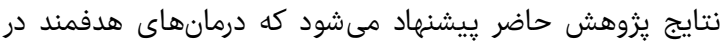

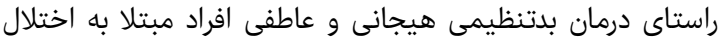

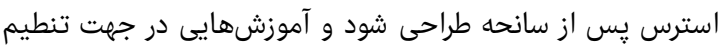

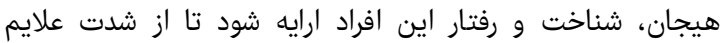

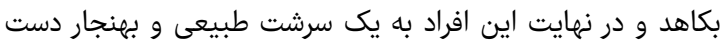

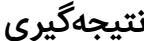

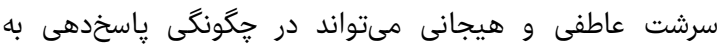

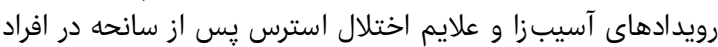

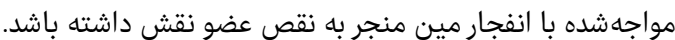

تشكر و قدردانى: از همكارى و همراهى صميمانه بنياد شهيد

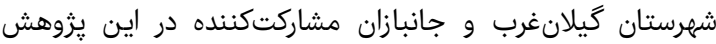
صميمانه سياسگزارى مى شودان

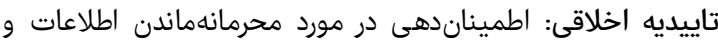

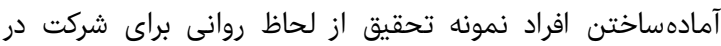

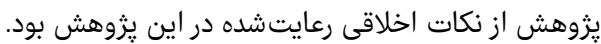

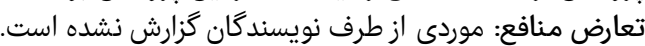

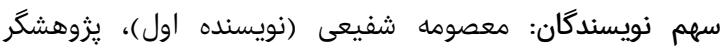

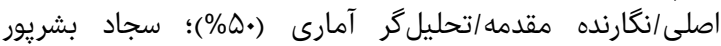

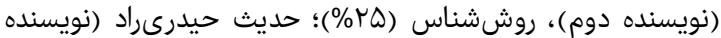

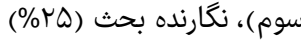

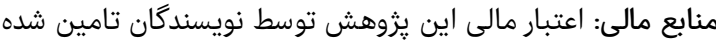

1- Beals I, Manson SM, Shore JH, Friedman M, AshcraftM, Fairbank JA, et al. The prevalence of posttraumatic stress disorder among American Indian veterans: disparities and context. J Trauma Stress. 2002;15(2):89-97.

2- Hizli FG, Taskintuna N, Isikli S, Kilic C, Zileli L. Predictors of posttraumatic stress in children and adolescents. Child Youth Serv Rev. 2009;31(3):349-54

3- Bugg A, Turpin G, Mason S, Scholes C. A randomized controlled trial of the effectiveness of writing as intervention for traumatic injury patients at risk of developing stress disorder. Behav Res Ther. 2009;47(1):6-12.

4- American Psychiatric Association. Diagnostic and statistical manual of mental disorders. $5^{\text {th }}$ edition. Washington DC: American Psychiatric Association; 2013. 5- Chossegros L, Hours M, Charnay P, Bernard M, Fort E, Boisson D, et al. Predictive factors of chronic posttraumatic stress disorder 6 months after aroad traffic accident. Accid Anal Prev. 2011;43(1):471-7.

6- Shafiee-Kamalabadi M, Bigdeli I, Alavi K, Kianersi F. Prevalence of post traumatic stress disorder and comorbid personality disorders in the groups veterans Tehran City. J Clin Psychol. 2014;6(1):65-75. [Persian]

7- Mandani B, Fakhri A. Study of health related quality of life in posttraumatic stress disorder war veterans. Iran J War Public Health. 2013;5(2):18-25. [Persian]

8- Rettew DC, McKee L. Temperament and its role in developmental psychopathology. Harv Rev Psychiatry. 2005;13(1):14-27.

9- Shafiei M, Basharpoor S, Ahmadi S, Heidarirad H. The comparison of temperament, character, and distraction

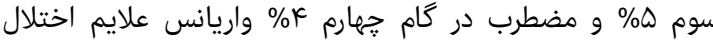

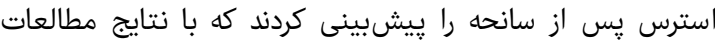

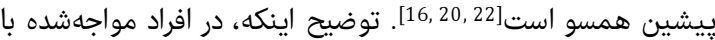

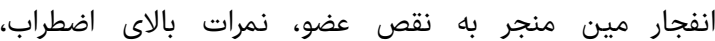

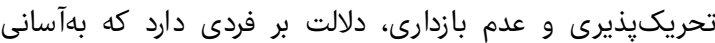

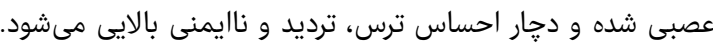

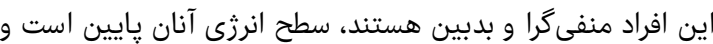

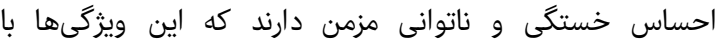

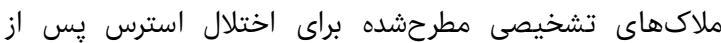

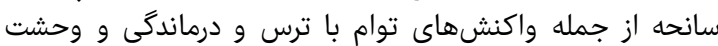

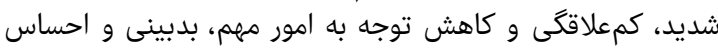

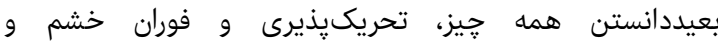
كوشبهزنكى همراستا است] [4].

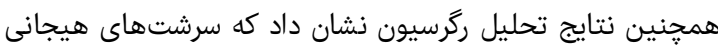

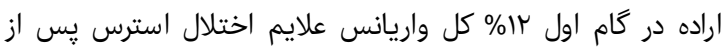

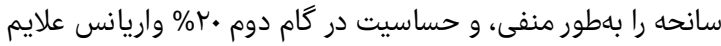

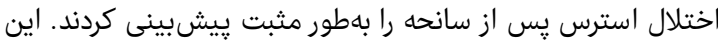

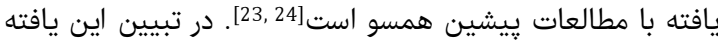

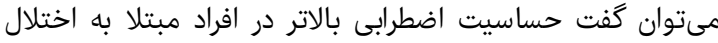

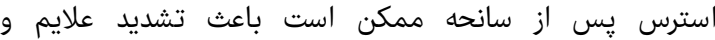

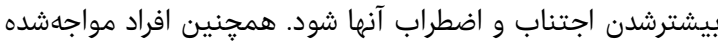

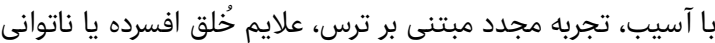

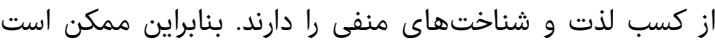

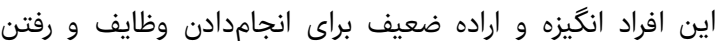

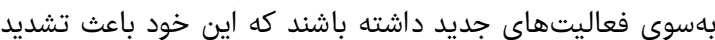

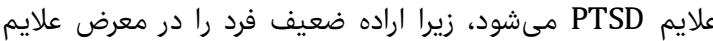

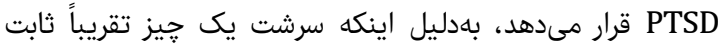

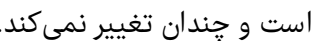

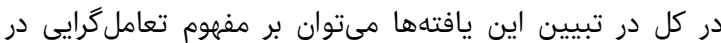

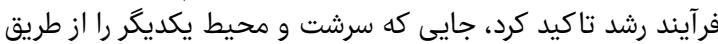

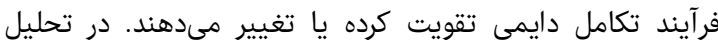

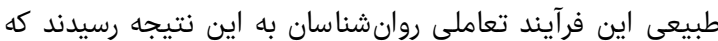

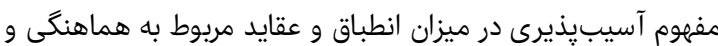

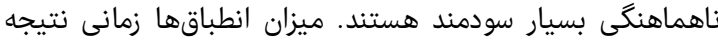

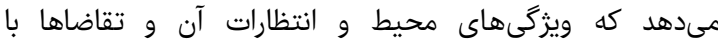

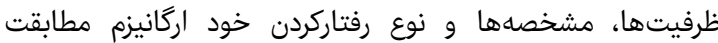

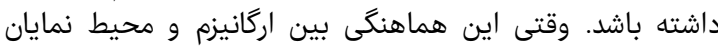

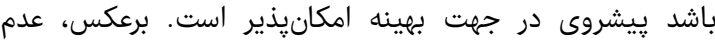

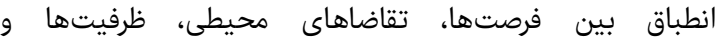

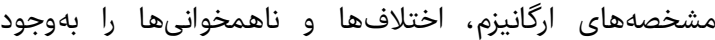
مى آورد، بهطورى كه رشد منحرف شده و عملكرد ناسازكارانه ييش بيش

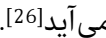

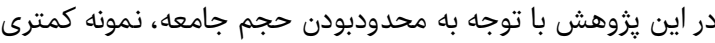

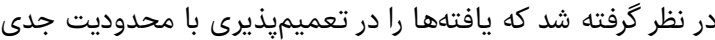

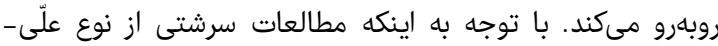

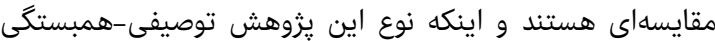
است و نيز ناتوانى در كنترل برخى متغيرهاى مزاحم جون إنى استفاده

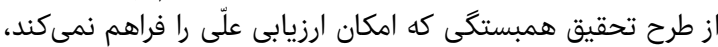

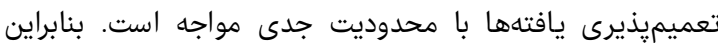

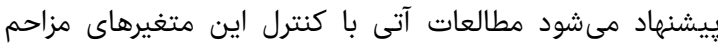

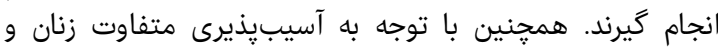

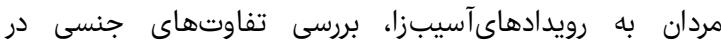




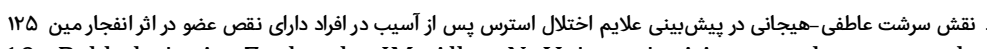

19- Bakhshaie A, Zvolensky JM, Allan N, Vujanovic AA, Schmidt BN. Differential effects of anxiety sensitivity components in the relation between emotional nonacceptance and post-traumatic stress symptoms among trauma-exposed treatment-seeking smokers. Cogn Behav Ther. 2015;44(3):175-89.

20- Spinhoven P, Penninx WB, van Hemert AM, de Rooij M, Elzinga MB. Comorbidity of PTSD in anxiety and depressive disorders: Prevalence and shared risk factors. Child Abuse Negl. 2014;38(8):1320-30.

21- Woodward SH, Shurick AA, Alvarez J, Kuo J, Nonyieva Y, Nonyieva Y, et al. A psycho physiological investigation of emotion regulation in chronic severe posttraumatic stress disorder. Psychophysiology. 2015;52(5):667-78.

22- van Rooij JS, Rademaker RA, Kennis M, Vink M, Kahn $\mathrm{SR}$, Geuze E. Impaired right inferior frontal gyrus response to contextual cues in male veterans with PTSD during response inhibition. J Psychiatry Neurosci. 2014;39(5):330-8.

23- Olatunji BO, Armstrong T, Fan Q, Zhao M. Risk and resiliency in posttraumatic stress disorder: Distinct roles of anxiety and disgust sensitivity. Psychol Trauma Theory Res Pract Policy. 2014;6(1):50-5.

24- Duffy RD, Jadidian A, Douglass RP, Allan BA. Work volition among U.S. veterans: Locus of control as a mediator. Couns Psychol. 2015;43(6):369-75.

25- Delavar A. The theorical and practical fundamental of research in social and human science. Tehran: Roshd Publication; 2004. p. 181. [Persian]

26- Keane TM, Caddell TM, Taylor L. Mississippi scalefor combay PTSD. J Consult Clin Psychol. 1998;56(1):85-90.

27- Goodarzi MA. Evaluating reliability and validity of the Mississippi scale for post-traumatic stress disorder in Shiraz. J Psycholo. 2003;7(2):153-78. [Persian]

28- Atkinson R, Nolen-Hoeksema S, Bem DJ, Smith EE, Atkinson RC. Hilgard's introduction topsychology. Zamani R, Bik M, Birshak B, Barahani MN, Shahraray M, translators. Tehran: Roshd Publication; 2016. p. 212. [Persian]

29- Schwind J, Gropalis M, Witthoft M, Weck F. The Effects of attention training on health anxiety: An experimental investigation. Cogn Ther Res. 2016;40(2):245-55.

30- Pakaslahti L. Children's and adolescents' aggressive behavior in context: the development and application of aggressive problem-solving strategies. Aggress Violent Behav. 2000;5(5):476-90.

between deaf and normal students. Educ Res J. 2017;3(33):61-74. [Persian]

10- Ali Malaeyri N, Kaviyani H, Asadi SM. Evaluation of personality dimensions using the Cloninger Temperament and Character Inventory in subjects with borderline personality disorders. Tehran Univ Med J. 2008;66(9):633-8. [Persian]

11- Lara DR, Bisol LW, Brunstein MG, Reppold CT, de Carvalho HW, Ottoni GL. The affective and emotional composite temperament (AFECT) model and scale: A system-based integrative approach. J Affect Disord. 2012;140(1):14-37.

12- Evans LM, Akiskal HS, Greenwood TA, Nievergelt CM, Keck Jr, PE, McElroy SL, et al. Suggestive linkage of a chromosomal locus on 18p11 to cyclothymic temperament in bipolar disorder families. Am J Med Genet B Neuropsychiatr Genet. 2008;147(3):326-32.

13- Perugi G, Toni C, Maremmani I, Tusini G, Ramacciotti $S$, Madia A, et al. The influence of affective temperaments and psychopathological traits on the definition of bipolar disorder subtypes: A study on bipolar I Italian national sample. J Affect Disord. 2012;136(1-2):41-9.

14- Brown WJ, Bruce SE, Buchholz KR, Artime MT, Hu M, \& Sheline IY. Affective Dispositions and PTSD Symptom Clusters in Female Interpersonal Trauma Survivors. J Interpers Violence. 2014; 5(1):17-39.

15- Mercer GT, Molinari V, Wright K, Pinnell C, Amin K, Sadek R. A proposed model of Alzheimer's dementia and PTSD: Pathophysiological processes in coping with traumatic negative affect. J Alzheimer Assoc. 2010;6(4):S494.

16- Armour C, Elhai JD, Richardson D, Ractliffe K, Wang L, Elklit A. Assessing a five factor model of PTSD: Is dysphoric arousal a unique PTSD construct showing differential relationships with anxiety and depression?. J Anxiety Disord. 2012;26(2):368-76.

17- Thomas KM, Hopwood CJ, Donnellan MB, Wright AG, Sanislow CA, McDevitt-Murphy ME, et al. Personality heterogeneity in PTSD: Distinct temperament and interpersonal typologies. Psychol Assess. 2014;26(1):2334.

18- Carmassi C, Stratta P, Massimetti G, Bertelloni AC, Conversano C, Cremone MI, et al. New DSM-5 maladaptive symptoms in PTSD: Gender differences and correlations with mood spectrum symptoms in a sample of high school students following survival of an earthquake. Ann Gen Psychiatry. 2014;13:28. 